\title{
SURFACE RESPONSE-BASED BEHAVIORAL MODELING OF ACCURATE DIGITIZERS: A CASE STUDY ON A FAST DIGITAL INTEGRATOR AT CERN
}

\author{
P. Arpaia ${ }^{1}$, V. Inglese ${ }^{2,3}$, G. Spiezia ${ }^{2,3}$, S. Tiso
}

\begin{abstract}
A statistical approach to behavioral modeling for assessing dynamic metrological performance during the concept design of accurate digitizers is proposed. A surface-response approach based on statistical experiment design is exploited for avoiding unrealistic hypothesis of linearity, optimizing simulation, exploring operating conditions systematically, as well as verifying identification and validation uncertainty. An actual case study on the dynamic metrological characterization of a Fast Digital Integrator for high-performance magnetic measurements at the European Organization for Nuclear Research (CERN) is presented.
\end{abstract}

1 University of Sannio, Department of Engineering, Benevento, Italy

2 University of Naples, Department of Engineering, University of Naples, Federico II, Via Claudio, Napoli, Italy 3 CERN, Accelerator Technology Department, Geneva, Switzerland.

To be published in the IMTC 2007 Special Issue of IEEE Transactions on Instrumentation and Measurement 


\title{
Surface Response-based Behavioral Modeling of Accurate Digitizers: A Case Study on a Fast Digital Integrator at CERN
}

\author{
Pasquale Arpaia ${ }^{1}$, Vitaliano Inglese $^{2}$, Giovanni Spiezia ${ }^{2}$, Stefano Tiso ${ }^{1}$ \\ ${ }^{1}$ Department of Engineering, University of Sannio, Corso Garibaldi 107, 82100 Benevento, Italy. \\ Ph : +39 0824 305804-17, Fax:+390824 305840, E-mail: arpaia@unisannio.it, Stefano.Tiso@cern.ch \\ ${ }^{2}$ Department of Engineering, University of Naples, Federico II, Via Claudio, Napoli, Italy; \\ CERN, Dept. AT (Accelerator Technology), Group MTM, CH 1211, Genève 23, Switzerland. \\ Ph : +41 2276 76635, Fax: +41 2276 76230, E-mail: \{Vitaliano.Inglese-Giovanni.Spiezia\}@cern.ch
}

\begin{abstract}
A statistical approach to behavioral modeling for assessing dynamic metrological performance during the concept design of accurate digitizers is proposed. A surface-response approach based on statistical experiment design is exploited for avoiding unrealistic hypothesis of linearity, optimizing simulation, exploring operating conditions systematically, as well as verifying identification and validation uncertainty. An actual case study on the dynamic metrological characterization of a Fast Digital Integrator for high-performance magnetic measurements at the European Organization for Nuclear Research (CERN) is presented.
\end{abstract}

Index term - Analog-to-digital converter (ADC), modeling, error analysis, statistics.

\section{INTRODUCTION}

In digital instrument design, metrological analysis by model simulation plays a key role during project assessment and enhancement [1]. Several indexes of metrological performance have to be evaluated in frequency and time domains (e.g., signal-to-noise-ratio, total harmonic distortion, integral and differential nonlinearity). This has to be carried out systematically, at varying operating conditions, influence parameters, and uncertainty sources, as well as, moreover, over the input range as a whole. During the conceptual design, behavioral modeling is useful for the sake of the generalization [1]: the digitizing chain is characterized by input-output analytical or numeric relations, without going in deep into the physical structure. In this way, a general perspective is kept, useful for several applications, owing to its independence on the specific architecture of the instrument and on its physical realization. 
Once the model is defined, simulations are carried out usually by following an intuitive approach: the model parameter's space is investigated by either or an One-Factor-At-Time strategy (OFAT) [2]-[3], or by a purely random variation. This can lead to misleading results, because (i) only a subspace of working conditions under investigation is explored and, (ii) moreover, often the digitizing chain is not appreciably linear and parameter interactions is not revealed in this way. Another more comprehensive approach is based on Monte-Carlo method in order to explore model parameter's space as a whole [4]: the instrument model is set and run in each possible configuration in order to have a total comprehension of the performance landscape. However, for high-performance accurate instruments, this technique turns out to be burdensome from a computational point of view [5]: in fact, the simulation burden depends on the number of parameters, the width of their working range, and the target accuracy of the analysis.

Experiment design techniques optimizes test burden in model identification by allowing a systematic exploration of a multidimensional parameter space with a high degree of resource exploitation [2][3]. Although mainly developed for physical runs, their use in simulation presents several improvement opportunities difficult or impossible in physical experiments [6]. In particular, Response Surface-modeling [8]-[9] allows statistical a-posteriori behavioral modeling [1]. More recent Multiple Response Surface (MRS) techniques provide better estimation of the local function nonuniformity (though biased) [9]. Conversely, Single Response Surface (SRS) methods assess the total nonuniformity (deterministic and random), more suitable for behavioral modeling.

In this paper, a behavioral modeling strategy, based on a Single Response Surface method, for tuning the simulation effort to the model complexity and the identification accuracy is proposed. In Section II, the proposed approach and the related model are illustrated. In Section III, a case study of a Fast Digital Integrator [7] for accurate dynamic magnetic measurements at European Organization for Nuclear Research (CERN) is shown.

\section{THE PROPOSAL}

In the following, (i) the basic ideas, (ii) the digitizer model, and (ii) the procedure of the proposed modeling approach are illustrated.

\section{A. Basic ideas}

The proposed approach exploits the following basic ideas: 
1. Behavioral architecture-independent modeling based on experiment design: Simulations have to be carried out for each $i$-th metrological performance index $q_{i}$ of $\mathbf{q}^{T}=\left(q_{1}, \ldots, q_{z}\right)$, which is in general a function $f_{i}: q_{i}=f_{i}(\mathbf{x}, \mathbf{c}, \mathbf{n})$ of (i) an input array $\mathbf{x}^{T}=\left(x_{1}, \ldots, x_{l}\right)$, (ii) the design parameters $\mathbf{c}^{T}=\left(c_{1}, \ldots, c_{m}\right)$ of the instrument, and (iii) the inner and outer error sources $\mathbf{n}^{T}=\left(n_{1}, \ldots, n_{h}\right)$. The experiment design-approach exploits an algebraic regression-model assumption about the way $\mathbf{x}, \mathbf{c}$, and $\mathbf{n}$ affect $\mathbf{q}$. Such a regression model $f_{i}: q_{i}=f_{i}(\mathbf{x}, \mathbf{c}, \mathbf{n})$ is an approximation of the digitizer model, thus it is a "model of a model", i.e. a metamodel. For this reason, it is generic and application independent, and intrinsically behavioral.

2. A-posteriori single response-surface approach to modeling: Model parameters are estimated by (i) making simulation runs at various input values for the $f_{i}$ domain $D: v=\left\{v_{1}, \ldots, v_{p}\right\}, \quad p=l+m+h, \quad$ representing the discretization of the $s$-dimensional simulation space, (ii) recording the corresponding responses, and then (iii) using standard leastsquares regression to estimate the coefficients. In particular, a Single Response Surface approach allows a detailed outlook of a single performance to be obtained, without pushing too much on a synthetic viewpoint of all the performance indexes.

3. Comprehensive investigation of operating conditions by means of statistical experiment planning: The $f_{i}$ domain $D$ is explored systematically by means of a statistical experiment planning [2]-[3]: given the model $f_{i}$, the optimum set of experiments for identifying such a model within a prefixed identification uncertainty, is found. The optimality is defined in terms of experiment position inside the simulation space $D$, as well as of number of experiments for achieving the prefixed uncertainty.

4. Nonlinear metamodel by interaction-based representation: Superposition effect-based models brings to misleading results in metrological analysis of high-performance instruments owing to the implicit linearity assumption: for this reason a twofold levels approach is exploited; a first screening attempt by means of a linear model, and, then, if necessary, a more complex. interaction-based model is utilized [2]-[3].

5. Metamodel accuracy assessment by statistical analysis of variance: the identification uncertainty of the metamodel is assessed by means of Multivariate ANalysis Of VAriance (MANOVA). 
6. Reliable metamodel identification and validation by statistical decision making: the metamodel identification and validation are assessed by means of statistical tests; in particular, (i) for the identification, the Fisher or Pillai's trace tests [11]-[12] are used for their robustness to mismatch of underlying hypotheses (especially for Fisher test), and (ii) for the validation, a $t$ test is employed in order to assess the significance of the metamodel prediction with reference to an independent experiment set.

\section{B. Digitizer model}

A behavioral model of a generic actual digitizer for metrological applications is shown in Fig. 1. The input signal passes through a programmable gain amplifier (PGA). The overall acquisition uncertainty is modeled as an additive random noise of standard deviation $\sigma_{V}$, and the overall acquisition distortion by a nonlinear deterministic block. Then, the amplified signal is digitized by an ideal analog-to-digital converter (ADC), introducing quantization and sampling noises. The time base is affected by an additive jitter noise of standard deviation $\sigma_{T}$. For the sake of generalization, a Universal Time Counter (UTC) can be inserted in order to have an absolute ADC-independent time reference for accurate time-dependent measurements. Finally, processing uncertainty is modeled as an additive numerical noise $n(t)$.

After a metrological test on the instrument (e.g. carried out according to IEEE Standard [13]), the performance $\mathbf{q}$ is determined.

\section{Procedure}

On the basis of the above basic ideas and model, a procedure for analyzing the metrological performance of a digitizer design is proposed. It is based on 3 main steps: (i) metamodel definition, (ii) metamodel identification, and (iii) metamodel validation (Fig. 2).

Metamodel Definition. As a first step, the metamodel inputs and parameters $\mathbf{x}, \mathbf{n}$, and $\mathbf{c}$ are defined (parameter definition) (Fig. 2) according to the most critical aspects of the digitizer design. By referring to the behavioral model in Fig. 1, the digitizer error sources $\mathbf{n}$ are (i) random, the voltage noise of the acquisition chain $\sigma_{V}$ and the time base jitter $\sigma_{T}$, and (ii) deterministic, the nonlinearity 
of the acquisition chain, modeled by suitable parameters. The digitizer design settings c consists of the use or not of the UTC, and the type of the processing algorithm, to be defined according to their impact on the performance. The digitizer performance index $\mathbf{q}$ is selected according to the specific quality aspect of the instrument to be investigated. They are mainly metrological indexes, static and dynamic, in the amplitude (e.g. DNL, or INL), time (e.g. stability), and frequency (e.g. signal-tonoise ratio) domains [13], but also functional performance, such as efficiency (e.g. throughput).

The representation models of the parameters are identified experimentally by determining in particular the variation range of each array component (experimental parameter identification). In this step, an estimation of the central values of the working ranges for each parameter is obtained, often by means of several identification techniques [14]. Once the components of the arrays $\mathbf{x}$, $\mathbf{c}$, and $\mathbf{n}$ are defined, they represent the continuous domain of the metamodel function $f$. According to the particular application, it has to be made discrete suitably, in order to carry out the simulations in a finite number of steps.

By following the above-mentioned a-posteriori approach, the order of the metamodel capable of describing suitably the digitizer metrological behavior is to be decided. In the practice of experimental design, combinatorial increase of the problem dimensionality is faced by means of a full quadratic model [11]. The function $\mathbf{q}=\mathbf{f}(\mathbf{x}, \mathbf{c}, \mathbf{n})=\mathbf{f}(\mathbf{v})$, describing the dependence of the performance on input signal, design setting parameters, and uncertainty sources, can expressed for the $i$-th index as :

$q_{i}=f_{i}(\mathbf{v})=a_{0 i}+\sum_{1 \leq k \leq p} b_{i k} v_{k}+\sum_{1 \leq k \leq p} \sum_{1 \leq j \leq p} d_{i k j} v_{k} v_{j}+\varepsilon$

where $\varepsilon$ represents the metamodel uncertainty and $a_{0 i}, b_{i k}$, and $d_{i k j}$ the coefficients of the corresponding response surface.

However, such a model turns out to be too heavy, thus a first-screening attempt based on a linear model is usually carried out:

$q_{i}=f_{i}(\mathbf{v})=a_{0 i}+\sum_{1 \leq k \leq p} b_{i k} v_{k}+\varepsilon$

Metamodel Identification. Afterwards, the coefficients of the response surface described by the model (1) - (2), best fitting of simulation and experimental data, have to be determined.

The suitability of the function $\mathbf{f}$ is verified by: (i) the simulation planning and running, (ii) the Analysis of Mean (ANOM), and (iii) the ANalysis Of VAriance (ANOVA). Then, an identification test 
is carried out in order to validate the surface-response metamodel.

$i$. Simulation planning and running. The optimum subset of the simulation domain $D$ is defined by selecting an experimental plan, according to the number of parameters and their levels, as well as the desired resolution [2], i.e. the desired information about covariance between the parameters. Then, the simulations are run according to the defined plan.

ii. ANOM. The main effects of each parameter on the mean response $\mu$ are assessed. In particular, the actual mean of the simulation runs is estimated, thus the main effect of the $k$-th parameter in any configuration $r$ can be evaluated as: $\delta_{k r}=m_{k r}-\mu$, where $m_{k r}$ is the mean of $q$ in all the runs where the k-th parameter is in the configuration $r$ (i.e., one of the points of the discrete domain $D$ ).

iii. ANOVA. The significance of the parameter effects is determined. In particular, ANOVA is aimed at determining, within a prefixed uncertainty, if a variation over the mean performance imposed by a corresponding parameter variation is due to the parameter itself or it can be confused with the model uncertainty.

Then, an identification test is carried out in order to check the model adequacy. If the performance index $\mathbf{q}$ is a scalar, the Fisher test can be used to establish the global parameter F-statistic of the model [10]. If $\mathbf{q}$ is an array, the Fisher test can be also applied to each component. A multicomponent check is carried out by determining the F-statistic through the Pillai's trace [12].

Metamodel validation. The metamodel is validated through Simulation Validation and Experimental Validation (Fig. 2).

The Simulation Validation aims at verifying the goodness of the simulator in itself, as well as of the identified metamodel. Performance is evaluated by simulations in points of the domain $D$, not considered in the simulation plan. The result is then compared with the metamodel prediction.

The Experimental Validation is based on the same concept: performance of an actual prototype of the instrument (or of a low-level simulator) in particular working points are measured and then compared with the metamodel forecasts. To validate the metamodel, the results must be consistent within to the uncertainty $\varepsilon$ of the metamodel. 


\section{EXPERIMENTAL CASE STUDY OF A FAST DIGITAL INTEGRATOR AT CERN}

The proposed approach was validated during the design of the Fast Digital Integrator (FDI) [7] in the laboratory of Magnet Test \& Measurement Group (Accelerator Technologies Dept.) at CERN.

In the following, (i) the FDI conceptual design, (ii) the metamodel definition, (iii) the metamodel identification, (iv) and the metamodel validation are illustrated.

\section{A. FDI Conceptual Design}

At CERN, a Fast Digital Integrator has been proposed for a real-time on-board integration of magnetic transducer signals [7]. In its conception, this instrument can digitize voltage signals over a bandwidth larger than state-of-art solutions, with a higher accuracy, owing to its 18 bit-resolution and $500 \mathrm{kS} / \mathrm{s}$-rate digital conversion, as well as with higher precision, owing to its self-correction of offset and gain errors.

In the FDI architecture, the input signal is conditioned by a low-noise low-distortion custom differential PGA, specifically designed at CERN. Then, it is digitized by an 18-bit SAR ADC. The ADC data are processed by means of a DSP, by exploiting external trigger pulses. At each pulse event, the results of the process is released. These pulse events are measured by a time-base with a resolution of $50 \mathrm{~ns}$ (UTC) in order to provide a fine link between the time and the angular domain. The processor supervisor of the FDI is the DSP. A field-programmable gate array (FPGA) acts as I/O processor of the DSP: it supervises the PGA operations at low level and provides the interface for the board bus PXI.

As an example, in a first application of magnetic flux measurement (test technique of rotating coil [16]), FDI has to sample the voltage arising from a coil transducer and to output the computed integral at a trigger frequency determined by an encoder, mounted on the shaft of the coil transducer rotating inside the superconductive magnet under test.

\section{B. Metamodel Definition}

In the FDI conceptual project, the design team is interested in investigating comprehensively and systematically the impact of possible nonideality sources $(\mathbf{n})$ on the dynamic distortion (q) of the FDI output, at varying the working operating conditions, mainly defined by the measurand $(\mathbf{x})$ and by the available design settings ( $\mathbf{c}$ ). 
Initially, under the assumption that the parameters $\mathbf{n}, \mathbf{x}$, and $\mathbf{c}$, are statistically independent, a firstorder screening metamodel is exploited in order to describe the dependency of $\mathbf{n}, \mathbf{x}$, and $\mathbf{c}$ on $\mathbf{q}$ (Eq. 2). If such an assumption should turn out to be inadequate after the validation, a second-order more accurate metamodel, including also interactions among parameters, is to be considered (Eq. 1).

In the design analysis, the team is interested in analyzing the impact of nonideality parameters and design settings on dynamic performance, at varying the input dynamic. For this reason, the input $\mathbf{x}$ of the FDI metamodel (Fig. 3) is expressed as Over-Sampling Ratio (OSR), i.e. the ratio between the ADC sampling rate $f_{S}$ and the trigger frequency $f_{t}$. As a matter of fact, a sample of processed output signal (i.e. the flux increment) is released at each trigger event, giving rise to a decimation.

In particular, the resulting nonideality of the FDI output as a whole has to be analyzed, thus the metamodel output $\mathbf{q}$ is the SIgnal-to-Noise And Distortion ratio (SINAD) of the computed integral.

At this stage of conceptual design, the team is interested in assessing the need for

(i) integration algorithms (e.g. trapezoidal) more advanced than the basic rectangular one. The team suspects that an increase in algorithm complexity, producing a loss in on-line computation time, could not produce a corresponding significant improvement in performance;

(ii) the possibility of using the UTC for a fine link between angular and time domains.

Therefore, the design settings parameters c are the type of integration algorithm (rectangular or trapezoidal), and the possibility of using the UTC (algorithm and timebase, respectively, in Fig. 3).

In the FDI acquisition and processing chain, main significant nonideality sources (n) are (Fig. 3) [1]: the overall acquisition amplitude random noise (modeled as additive [1] and expressed as its standard deviation $\sigma_{V}$ ), the time stamp jitter noise (in terms of its standard deviation $\sigma_{T}$ ), and the deterministic nonlinearity of the input/output characteristic. At this last aim, Kim proposed a meaningful model [17], relating the characteristic shape to the FFT test results: in particular, a parameter $\chi$ takes into account the asymmetry of the characteristic, while a parameter $\xi$ its exponential nonlinearity.

By summarizing, the parameter arrays of the FDI metamodel are:

$x=\mathrm{OSR} ; q=\mathrm{SINAD} ; \mathbf{c}^{T}=($ Algorithm, $\mathrm{UTC}) ; \mathbf{n}^{T}=\left(\sigma_{T}, \sigma_{V}, \chi, \xi\right)$.

The working range of each parameter and its levels are determined according to the following procedures: 
- OSR: by assuming an ADC sampling rate of $125 \mathrm{kS} / \mathrm{s}$, three different values of OSR were chosen, $(1,12.5$, and 125) in order to evaluate the performance for the different values of trigger frequency $\left(f_{t}\right), 125 \mathrm{kHz}, 10 \mathrm{kHz}$, and $1 \mathrm{kHz}$.

- Time stamp jitter: it was defined through the reference clock data sheet [18].

- Acquisition noise: by assuming a Gaussian distribution, the acquisition noise was derived experimentally, according to:

$$
\begin{aligned}
& \mathrm{SNR}=10 \cdot \log \frac{P_{\text {sig }}}{P_{\text {noise }}}=10 \cdot \log \frac{P_{\text {sig }}}{\sigma_{V}^{2}} ; \\
& \sigma_{V}^{2}=\frac{P_{\text {sig }}}{10^{\left(\frac{\text { SNR }}{10}\right)}} ;
\end{aligned}
$$

where SNR is the Signal-to-Noise Ratio in $\mathrm{dB}$ (i.e. the SINAD without the harmonic distortion), $P_{s i g}$ is the input signal power, and $P_{n o i s e}$ is the noise power over all the bandwidth, expressed by the variance $\sigma_{V}^{2}$.

- ADC nonlinearity parameters: according to the abovementioned Kim's two-parameters ADC model [17], a direct relation between the second and third harmonics amplitude and $\xi$ and $\chi$ is derived from the FFT of the output:

$$
\begin{aligned}
& \chi \square 2 \frac{A m p_{I I}}{\left(A m p_{I}\right)^{2}} ; \\
& \xi \square 4 \frac{A m p_{I I I}}{A m p_{I}} ;
\end{aligned}
$$

where $A m p_{i}$ represents the amplitude of the $i$-th harmonic.

In Tab. I, the numerical values of the FDI metamodel parameters, selected according to the abovementioned criteria, are reported.

\section{C: Metamodel Identification}

According to the procedure of Fig. 2, the metamodel is identified by: (i) Simulation Planning, (ii) ANOM and ANOVA, and (iii) Fischer test. 
Simulation Planning: A Resolution-III standard Taguchi plan L18 is used [19], owing its capability of exploring a combinatorial space generated by up to seven 3-level parameters and one 2-level parameter, according to a first-order model.

A first simulation cycle showed that the UTC has a largely predominating influence on the performance, by making the other parameters negligible. Thus, the UTC was permanently used in the further simulation planning and the only design setting parameter left is the algorithm. In this case, one 2-level (integration algorithm type) and four 3-level (time stamp jitter, acquisition noise, $\xi$, and, $\chi$ ) parameters are considered, thus 8 degrees of freedom are left for determining the model uncertainty.

ANOM and ANOVA. In Tab. II, the effects of each parameter on the average SINAD are shown (ANOM results), for an OSR of 125 due to an ADC sampling rate of $125 \mathrm{kS} / \mathrm{s}$ and a trigger frequency equal to $1 \mathrm{kHz}$. By the table, a predominance of the non-linearity parameter $\xi$ and $\chi$ seems to rise up (especially of $\chi$ ), expressed in terms of effect range $\Delta$. Furthermore, the time stamp jitter and the acquisition noise have the largest influence on the SNR such as expected, because the non-linearity parameters affect the amplitudes of the harmonics, and, thus, their effects are not detectable by means of the SNR index.

Tab. III reports the ANOVA results, by showing (i) the contribution of the i-th parameter to the performance variance $\left(\sigma_{i}^{2}\right)$, (ii) the corresponding F-statistic $\left(\mathrm{F}_{i}\right)$, and (iii) the P-parameter $(P)$ pointing out the probability that a parameter does not affect $\mathbf{q}$, with a confidence level of $1 \%$.

The SINAD is a scalar quantity, thus the goodness of the first-order model was verified by a Fisher test. As larger the index $F_{i}$ is, as the variation over the mean is due to the parameter variation and not to the model uncertainty.

Thus, the F-statistic and the P-parameter of Tab. III show that the most significant parameter is the non-linearity parameter $\chi$. As a matter of fact, it is directly related to the harmonics of the signal to be integrated.

Actually, its effect cannot be avoided, but the proposed technique allowed the reachable improvement to be pointed out, if the FDI analog part is optimized, or if a suitable correction is carried out.

The Fisher test confirms the suitability of the first-order model to represent the FDI behavior, thus, the weight coefficient of the re-arranged metamodel was determined: 


$$
\operatorname{SINAD}=69.1+8.61 \cdot \tilde{\chi}
$$

where:

$$
\tilde{\chi}=\frac{\log (\chi)-\frac{\hat{\chi}_{\max }-\hat{\chi}_{\min }}{2}}{\hat{\chi}_{\max }}, \hat{\chi}_{\text {max }}=\log \left(\chi_{\text {max }}\right), \hat{\chi}_{\text {min }}=\log \left(\chi_{\min }\right),
$$

and $\chi_{\max }$ and $\chi_{\min }$ are the maximum and minimum value of the parameter $\chi$ respectively (Tab. I). Eq. 7 expresses the dependence of the SINAD index on the non-linearity parameter $\chi$, related to the harmonic distortion.

\section{Metamodel Validation}

In Fig. 4, the FDI performance in terms of SINAD is depicted for different value of the OSR. The graph shows that the predictions of the model are consistent with the simulated results, as well as with the experimental results within a model band of uncertainty of $1 \sigma$.

The model is highly helpful in determining the sensitivity of the FDI overall distortion to the ADC nonlinearity. Moreover, the results of model analysis point out that the most important distortion to be corrected is the asymmetry of the transfer function represented by $\chi$. This is a valuable requirement for the FDI correction procedure of the dynamic distortion to be realized.

\section{CONCLUSION}

In this paper, an error modeling technique of accurate digitizers for metrological purposes is presented. A systematic procedure allows the behavioral model of the device to be defined, identified, and validated in an efficient way.

As a case study, a Fast Digital Integrator, developed at CERN for magnetic measurements, was analyzed and modeled to understand its main uncertainty sources. The results show the practical usefulness of the proposed approach in defining the impact of uncertainty source on working conditions and design choices during the concept design of a digital instrument. As a matter of fact, the model pointed out that the most important distortion to be corrected is the asymmetry of the transfer function. This is an important indication for the FDI dynamic correction procedure to be realized. 


\section{ACKNOWLEDGMENTS}

This work was sponsored by CERN trough the agreement No. K 1201/AT/LHC with the Department of Engineering, University of Sannio, whose support authors gratefully acknowledge. Authors thank Philippe Lebrun, Director of CERN Department of Accelerator Technology, for his kind permission to the partial disclosure of research results, according to the article 5 of the above agreement and the related international laws. Authors thank also Luca Bottura, Felice Cennamo, Domenico Della Ratta, and Louis Walckiers for their useful cooperation. 


\section{REFERENCES}

[1] P. Arpaia, P.Daponte, S.Rapuano, "A state of the art on ADC modeling", Computer Standards and Interfaces, Vol. 26, , pp.31-42, 2003.

[2] G. E. P. Box, W. G. Hunter and J. Stuart Hunter, J. Wiley, Statistics for Experimenters: Design, Innovation, and Discovery, $2^{\text {nd }}$ Ed., New York, 2005.

[3] G. E. P. Box, N. R. Draper, J. Wiley, Empirical Model-Building and Response Surfaces, New York, 1987.

[4] P. W. A. Lewis, Simulation Methodology for Statisticians, Operations Analysts, and Engineers, McKenzie, 1988.

[5] P. Arpaia, R. Schiano Lo Moriello, "Metrological Characterization Based on a Computer Aided Experimental Design of a Frequency Response Analyzer", Proc. of ISCS2001, 6-7 December 2001, Naples, pp.150-161.

[6] W. D. Kelton, "Experimental Design for Simulation”, in Proc. of 2000 Winter Sim. Conf., ed. J.A. Joines, R.R. Barton, K. Kang, and P.A. Fishwick, 32-38. Piscataway, New Jersey: IEEE.

[7] P. Arpaia, A. Masi, G. Spiezia, "A Digital Integrator for Fast and Accurate Measurement of Magnetic Flux by Rotating Coils", IEEE Transactions on Instrumentation and Measurements, Vol. 56, No. 2, April 2007.

[8] Douglas C. Montgomery, Raymond H. Myers, Response Surface Methodology: Process and Product Optimization Using Designed Experiments, Wiley-Interscience, Jan. 1995.

[9] M. Papila, R. T. Haftka, "Response Surface Approximations: Noise, Error Repair, and Modeling Errors," AIAA Journal, Vol. 38, No. 12, Dec. 2000.

[10] T. H. Smith, B. E. Goodlin, D. S. Boning, H. H. Sawin, “A Statistical Analysis of Single and Multiple Response Surface Modeling”, IEEE Transactions on Semiconductor Manufacturing, Vol. 12, No. 4, pp. 419-430, Nov. 1999.

[11] D. E. Johnson, Applied Multivariate Methods for Data Analysis. Pacific Grove, CA, Duxbury Press.

[12] B. G. Tabachnick, L. S. Fidell, Using Multivariate Statistics, $4^{\text {th }}$ Ed. New York, Allyn \& Bacon. 
[13] IEEE Std 1057-1994. IEEE Standard for Digitizing Waveform Recorders.

[14] L. Ljung, "State of the Art in Linear System Identification: Time and Frequency Domain Methods", in Proc. of 2004 American Control Conf., Boston, MA, June 30 - July 2, 2004.

[15] R. Bellman, Dynamic Programming, Princeton University Press, Princeton, New Jersey, 1957.

[16] J. Billan, L. Bottura, M. Buzio, G. D’Angelo, G. Deferne, O. Dunkel, P. Legrand, A. Rijllart, A. Siemko, P. Sievers, S. Schloss, L. Walckiers, "Twin Rotating Coils for Cold Magnetic Measurements of 15m Long LHC Dipoles”, LHC Project Report 361, CERN, 1 Dec. 1999.

[17] K. Kim, "Analog-to-Digital Conversion and Harmonic Noises Due to the Integral Nonlinearity", IEEE Transactions on Instrumentation and Measurement, Vol. 43, No. 2, April 1994.

[18] http://www.microcrystal.com/productdocuments/variants/OCXOVT._6989.pdf

[19] D. K. J. Lin “Making full use of Taguchi's orthogonal arrays", Quality and Reliability Engineering International, Vol. 10, pp. 117-121, 1994. 


\section{LIST OF FIGURE CAPTIONS}

Fig. 1 Behavioral model of a generic actual digitizer.

Fig. 2 Procedure for metamodel-based analysis.

Fig. 3 FDI behavioral model and the metamodel parameters.

Fig. 4 Comparison among simulated performance, experimental performance, and metamodel predictions.

\section{LIST OF TABLE CAPTIONS}

Table I. Numerical values of the metamodel parameters.

Table II. ANOM results at $1 \mathrm{kHz}$ trigger frequency.

Table III. ANOVA results at $1 \mathrm{kHz}$ trigger frequency. 


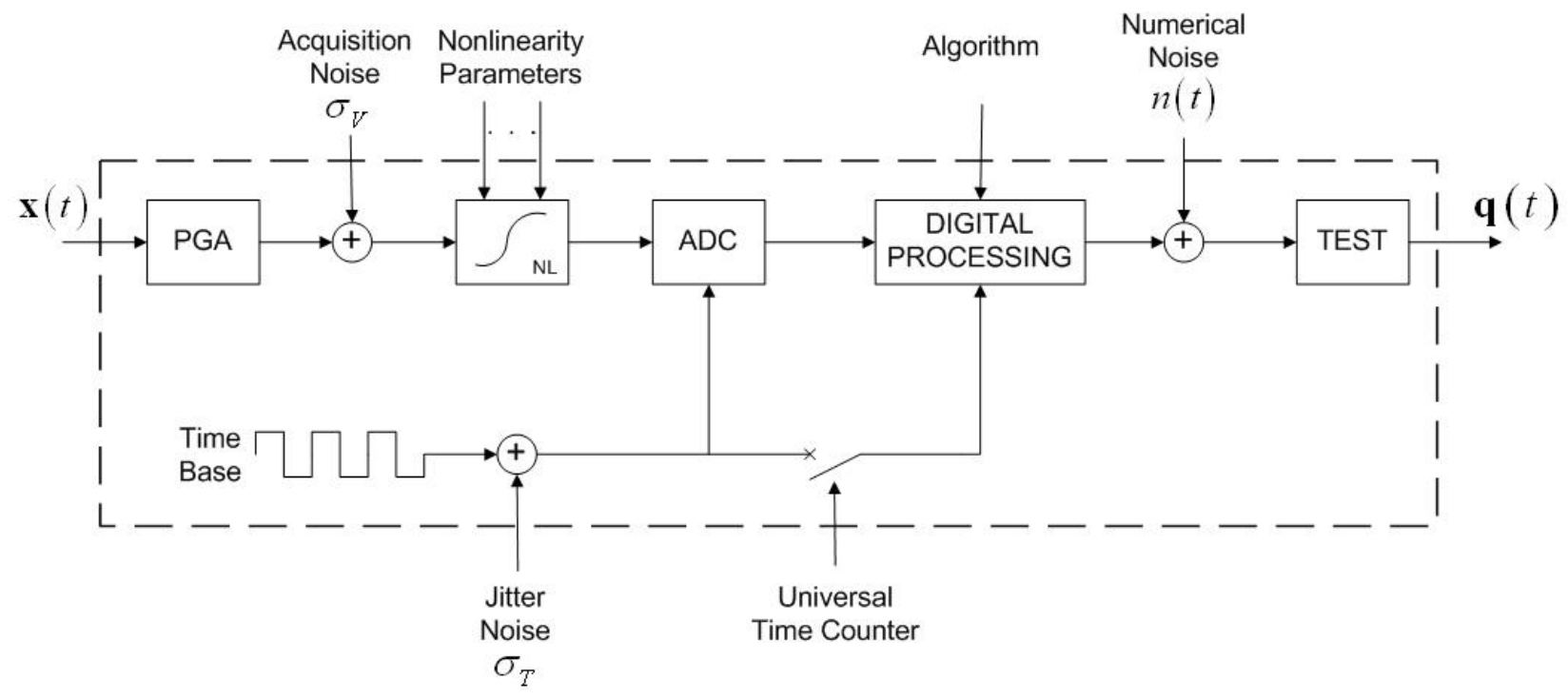

Fig. 1 Behavioral model of a generic actual digitizer. 


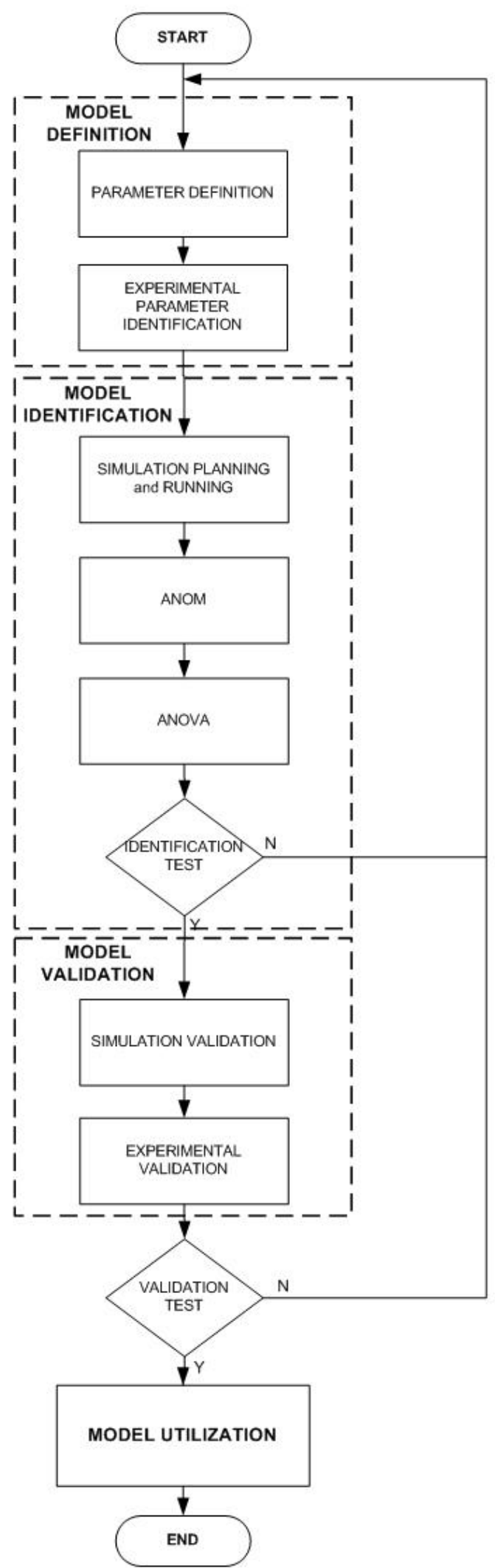

Fig. 2 Procedure for metamodel-based analysis. 


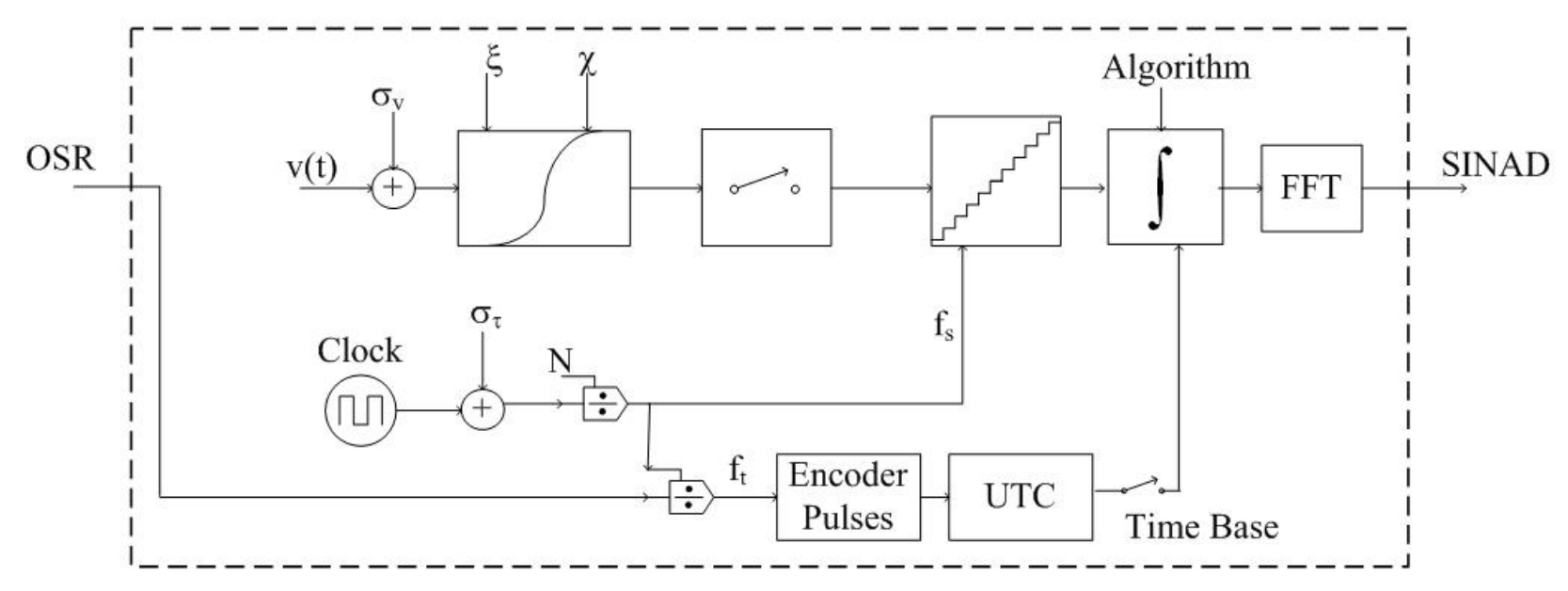

Fig. 3 . FDI behavioral model and the metamodel parameters.

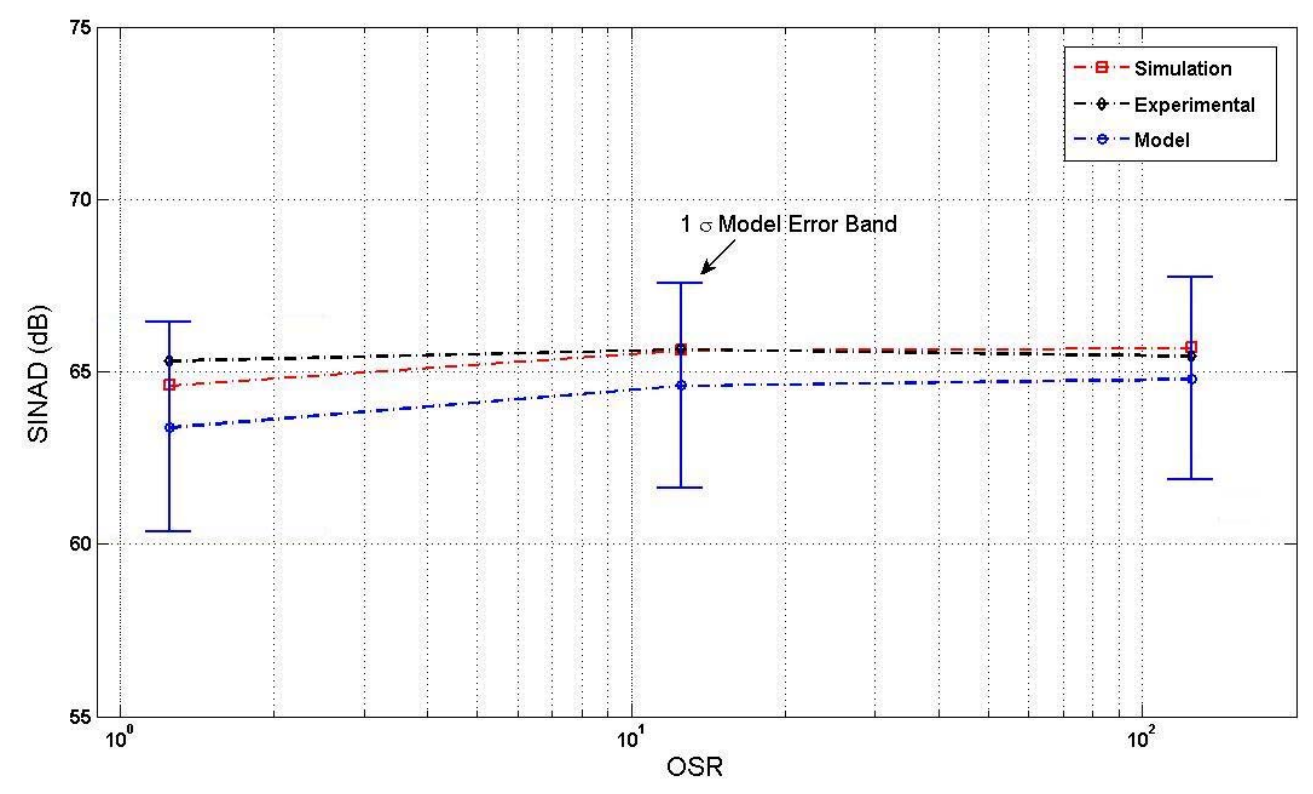

Fig. 4 Comparison among simulated performance, experimental performance, and metamodel predictions. 
Table I. Numerical values of the metamodel parameters.

\begin{tabular}{|c|c|c|c|}
\hline & \multicolumn{3}{|c|}{ Levels } \\
\hline & 1 & 2 & 3 \\
\hline Algorithm & rectangular & trapezoidal & \# \\
\hline Universal Time Counter & present & absent & \# \\
\hline Time Stamp Jitter & $5 \cdot 10^{-15}$ & $5 \cdot 10^{-13}$ & $5 \cdot 10^{-12}$ \\
\hline Acquisition Noise & $4.7 \cdot 10^{-4}$ & $3.7 \cdot 10^{-4}$ & $2.7 \cdot 10^{-4}$ \\
\hline$x_{2}$ & $5.0 \cdot 10^{-4}$ & $1.6 \cdot 10^{-4}$ & $5.0 \cdot 10^{-5}$ \\
\hline$\xi$ & $500 \cdot 10^{-6}$ & $250 \cdot 10^{-6}$ & $1 \cdot 10^{-6}$ \\
\hline
\end{tabular}

Table II. ANOM results at $1 \mathrm{kHz}$ trigger frequency.

\begin{tabular}{|l|c|c|c|c|}
\hline & \multicolumn{3}{|c|}{ Levels } \\
\hline & $\mathbf{1}$ & $\mathbf{2}$ & $\mathbf{3}$ & $\boldsymbol{\Delta}$ \\
\hline Algorithm & -0.01 & 0.01 & $\#$ & 0.01 \\
\hline Time Stamp Jitter & 0.32 & -0.16 & -0.16 & 0.49 \\
\hline Acquisition Noise & 0.31 & -0.15 & -0.16 & 0.47 \\
\hline $\boldsymbol{\chi}$ & -9.50 & 0.25 & 9.25 & 18.75 \\
\hline $\boldsymbol{\xi}$ & -0.78 & 0.31 & 0.46 & 1.24 \\
\hline
\end{tabular}

Table III. ANOVA results at $1 \mathrm{kHz}$ trigger frequency.

\begin{tabular}{|l|c|c|c|c|}
\hline & $\gamma$ & $\sigma_{1}^{2}$ & $\mathbf{F}_{\mathbf{i}}$ & $\mathbf{P}$ \\
\hline Time Stamp Jitter & 2 & $4,7 \cdot 10^{-1}$ & $7,0 \cdot 10^{-1}$ & $5,2 \cdot 10^{-1}$ \\
\hline Acquisition Noise & 2 & $4,3 \cdot 10^{-1}$ & $6,4 \cdot 10^{-1}$ & $5,5 \cdot 10^{-1}$ \\
\hline$\chi$ & 2 & $5,3 \cdot 10^{2}$ & $7,9 \cdot 10^{2}$ & $<10^{-4}$ \\
\hline$\xi$ & 2 & 2,7 & 4,1 & $6,0 \cdot 10^{-2}$ \\
\hline Algorithm & 1 & $5,0 \cdot 10^{-4}$ & $7,5 \cdot 10^{-4}$ & $>\left(1-10^{-4}\right)$ \\
\hline Error & 8 & $6,7 \cdot 10^{-1}$ & & \\
\hline
\end{tabular}

